\title{
Comparação da aptidão física de mulheres adultas e idosas de acordo com o histórico de quedas e a prática regular de diferentes modalidades de exercícios físicos
}

\author{
Comparison of the physical fitness of older adult women according to the \\ history of falls and the regular practice of different physical exercises
}

Mariana Rodrigues de Oliveira Reis ${ }^{1}$ (D), Javier Brazo-Sayavera² (D), Jhennyfer Aline Rodrigues Lima $^{3}$ (D), Jonatas Evandro Nogueira ${ }^{(1 D}$, Anderson Saranz Zago ${ }^{5}$ (D), Átila Alexandre Trapé 6 (D)

\begin{abstract}
RESUMO
Objetivos: Comparar a aptidão física de mulheres de acordo com o histórico de quedas e a prática regular de diferentes modalidades de exercícios físicos (treinamento combinado: musculação e resistência aeróbia; treinamento funcional: multicomponente; e treinamento multimodal: duas ou mais modalidades). Métodos: Estudo transversal com 44 mulheres (idade entre 50 e 80 anos). Foram coletados dados sociodemográficos e sobre o histórico de quedas no último ano, além da avaliação antropométrica e testes motores (flexibilidade, força de membros superiores e inferiores, capacidade aeróbia e agilidade e equilíbrio dinâmico) para avaliar a aptidão física. Na análise estatística, foram realizados o Teste t para amostras independentes e a ANCOVA utilizando idade, índice de massa corporal e tempo de exercício físico como co-variáveis. Resultados: As participantes que tiveram ocorrência de quedas no último ano apresentaram piores resultados nos testes de flexibilidade (sentar e alcançar e mãos nas costas), força de membros superiores (flexão de cotovelo) e de membros inferiores (sentar e levantar), bem como capacidade aeróbia (teste de caminhada de seis minutos). Em relação às diferentes modalidades de exercícios físicos, não foi possível observar diferença estatística para nenhuma das variáveis estudadas. Entretanto, resultados satisfatórios de acordo com os valores normativos foram observados nos três grupos. Conclusões: 0 grupo com histórico de quedas apresentou piores resultados na aptidão física. Em relação às diferentes modalidades de treinamento, não houve diferença entre os grupos para nenhuma variável analisada. Entretanto, observa-se que estas modalidades são importantes para a manutenção de bons níveis de aptidão física no contexto do envelhecimento.
\end{abstract}

Palavras-chave: Atividade Física; Envelhecimento; Idosos; Quedas.

\begin{abstract}
Objectives: Comparing women's physical fitness according to the history of falls and regular practice of different physical exercises (combined training: strength and aerobic training; functional training: multicomponent; and multimodal training: two or more styles). Methods: A cross-sectional study was conducted with 44 women (aged between 50 and 80 years old). Sociodemographic data and history of falls in the last year were collected, in addition to anthropometric assessment and motor tests (flexibility, upper and lower limb strength, aerobic capacity and agility, and dynamic balance) to assess physical fitness. In the statistical analysis, independent samples t-test and ANCOVA using age, body mass index, and time of physical exercise as covariates were performed. Results: The participants who had falls in the last year presented worse outcomes in the flexibility tests (sit and reach and hands-on-the back), strength tests of upper limbs (elbow flexion), and lower limbs (sit and stand up), as well as aerobic capacity (six-minute walking test). Regarding the different modalities of exercises, it was not possible to observe statistical differences for any of the variables studied. However, satisfactory results according to the normative values were observed in the three groups. Conclusions: The group with a history of falls had worse outcomes in physical fitness. Regarding the different modalities of training, there was no difference between the groups for any variable analyzed. However, it seems that these modalities are essential to maintain satisfactory levels of physical fitness in the context of aging.
\end{abstract}

Keywords: Physical Activity; Aging; Elderly; Accident Falls.

1. Profissional de Educação Física (Bacharel), Universidade Paulista (UNIP), Ribeirão Preto (SP), Brasil.

2. Docente no Polo Desarrollo Universitario Educación Física, Salud y Calidad de Vida. Centro Universitario Regional Noreste. Universidad de la República (UdelaR), Uruguay.

3. Postdoc do Laboratório de Fisiologia e Fisioterapia Cardiovascular da Faculdade de Medicina de Ribeirão Preto (FMRP), Universidade de São Paulo (USP), Ribeirão Preto (SP), Brasil.

4. Educador na Escola de Educação Física e Esporte de Ribeirão Preto (EEFERP), USP, Ribeirão Preto (SP), Brasil.

5. Docente no Departamento de Educação Física da Universidade Estadual Paulista (UNESP), Bauru (SP), Brasil.

6. Docente na Escola de Educação Física e Esporte de Ribeirão Preto (EEFERP), USP, Ribeirão Preto (SP), Brasil.

$\bowtie$ Átila Alexandre Trapé. Escola de Educação Física e Esporte de Ribeirão Preto (EEFERP). Avenida dos Bandeirantes, 3900, Campus Universitário - Bairro Monte Alegre. CEP: 14040-907. Ribeirão Preto (SP), Brasil. atrape@usp.br | Recebido em: 19/02/2020 | Aprovado em: 09/06/2020 


\section{INTRODUÇÃO}

As alterações nos aspectos físicos e fisiológicos do organismo e a maior vulnerabilidade para a incidência de doenças podem estar relacionadas ao envelhecimento, mas também ao estilo de vida sedentário adotado ao longo da $v^{v i d a}{ }^{1}$. Neste contexto, a perda de massa e força muscular e a diminuição da agilidade e da flexibilidade apresentam consequências prejudiciais na funcionalidade dos idosos, levando ao comprometimento de diversos sistemas orgânicos, em especial os relacionados ao equilíbrio, podendo causar quedas ${ }^{2,3}$.

A queda no idoso é um problema de Saúde Pública em termos de morbidade, mortalidade e custos para serviços sociais e de saúde ${ }^{4,5}$. O risco de quedas aumenta com a idade, podendo provocar fraturas e lesões, com consequente redução da independência e restrições das atividades de vida diária (AVDs), que ainda podem também estar relacionadas ao medo de cair ${ }^{6}$.

Por outro lado, o estilo de vida ativo pode minimizar os efeitos relacionados ao envelhecimento, promover uma melhor condição de saúde e diminuir a incidência de quedas ${ }^{3,7}$. Praticantes regulares de exercícios físicos podem reduzir em $61 \%$ o risco de fraturas, bem como diminuir em $43 \%$ a necessidade de cuidados médicos relacionados às quedas ${ }^{6}$. Desta forma, a participação em diferentes modalidades de exercícios físicos pode promover benefícios globais no organismo como melhora da composição corporal, agilidade, equilíbrio, resistência aeróbia, coordenação, flexibilidade, força e velocidade, atributos estes que compõem a aptidão física ${ }^{8,9}$. Assim, bons níveis de aptidão física estão relacionados à manutenção da capacidade funcional, levando à realização de AVDs de forma independente, interação com a comunidade, além da diminuição de riscos para doenças crônicas não transmissíveis ${ }^{2,7}$.

Diferentes modalidades de exercícios físicos são analisadas no presente trabalho: (i) treinamento combinado: combinação de treinamento de força (musculação) e treinamento de resistência aeróbia (intervalado ou contínuo: caminhada, corrida, bicicleta, natação, etc); (ii) treinamento funcional: atividade multicomponente na mesma sessão (força, resistência aeróbia, flexibilidade, equilíbrio, agilidade, entre outras); (iii) treinamento multimodal: combinação de duas ou mais modalidades na rotina de exercícios físicos que podem envolver o treinamento de musculação ou aeróbio, juntamente com outras modalidades como pilates (solo ou estúdio), ioga, alongamento, dança, entre outras. É possível observar que estas três modalidades de exercícios físicos apresentam em comum propostas globais com estímulos para os diversos atributos que compõem a aptidão física, assim como vem sendo preconizado por publicações importantes que contemplam a discussão sobre intervenções de exercícios físicos relacionadas ao processo de envelhecimento $^{9-11}$. Entretanto, cada uma delas apresenta particularidades no seu desenvolvimento já que o treinamento combinado envolve duas capacidades físicas que recebem maior destaque na literatura $^{12}$; o treinamento funcional pode envolver mais capacidades físicas, mas por ser multicomponente, pode não enfatizar o trabalho das capacidades de um forma mais específica; e, enquanto nos treinamentos combinado e funcional, o trabalho é realizado dentro de uma mesma sessão, no treinamento multimodal temos a combinação de diferentes tipos de exercícios físicos que são realizados separadamente ${ }^{13}$.

Este estudo parte do conhecimento de que o estilo de vida ativo está associado a menor ocorrência de quedas ${ }^{3,6}$, assim como indivíduos que praticam exercícios físicos regularmente apresentam bons níveis de aptidão física, de acordo com valores normativos disponíveis na literatura ${ }^{13-15}$. A partir disto, o aprofundamento em questões mais específicas pode ser importante para o conhecimento em atividade física, saúde e envelhecimento. Sendo assim, qual seria a diferença na aptidão física entre mulheres que apresentaram com as que não apresentaram ocorrência de queda no último ano? E, existe diferença na aptidão física de praticantes destas três modalidades de exercícios físicos que são desenvolvidas sob este ponto de vista mais global? Assim, o objetivo deste trabalho foi comparar a aptidão física de mulheres com idade entre 50 e 80 anos de acordo com o histórico de quedas e a prática regular de diferentes modalidades de exercícios físicos (treinamento combinado, treinamento funcional e treinamento multimodal). 


\section{MATERIAL E MÉTODOS}

\section{Tipo de Estudo e Casuística}

As coletas deste estudo transversal foram realizadas de janeiro a setembro de 2019 com 44 mulheres (50 a 80 anos) participantes dos programas de exercícios físicos da Associação Cristã de Moços de Ribeirão Preto (ACM-RP) e da Escola de Educação Física e Esporte de Ribeirão Preto (EEFERP) da Universidade de São Paulo (USP). Os critérios de inclusão foram: ser do sexo feminino, ter idade entre 50 e 80 anos e praticar uma das modalidades de treinamento (combinado, funcional ou multimodal) há pelo menos seis meses. Os critérios de exclusão foram: faltar em alguma avaliação ou apresentar alguma condição médica que pudesse impedir a realização dos testes motores.

O presente estudo foi aprovado pelo Comitê de Ética e Pesquisa (CEP) da Universidade Paulista (UNIP) mediante o protocolo de aprovação número 3.065.790 em 07/12/2018.

Alguns cuidados foram tomados visando a qualidade dos dados coletados pelo presente estudo: (i) discussão teórica e treinamento prático de todos os aspectos da avaliação: aplicação dos Instrumentos, testes motores e medidas antropométricas; (ii) o mesmo avaliador ficou responsável por realizar a mesma parte da avaliação em todas as participantes nos diferentes dias em que aconteceram as avaliações; (iii) a segunda tentativa dos testes motores que apresentavam esta possibilidade no seu protocolo foi realizada logo na sequência da primeira e pelo mesmo avaliador; (iv) foi realizado um estudo piloto com $n=10$ para verificar possíveis dificuldades e possibilitar os ajustes antes das coletas.

\section{Instrumentos}

As participantes responderam uma anamnese contendo informações pessoais, relacionadas ao tempo de prática de exercícios físicos, modalidades praticadas e sobre o estado de saúde (hipertensão, diabetes e dislipidemias). Além disso, para caracterizar a amostra, foram aplicados os questionários: (i) International Physical Activity Questionnaire (IPAQ) - versão curta, validado no Brasil e com reprodutibilidade determinada ${ }^{16}$ para estimar o nível de atividade física realizado regularmente (atividade física no trabalho, como locomoção em casa e no lazer); (ii) e o Critério Socioeconômico ${ }^{17}$, que apresenta forte sustentação teórica e metodológica, além de consistência em termos de validação ${ }^{18}$, para classificar economicamente cada participante, por meio da posse de bens materiais, grau de instrução do chefe da família e acesso a serviços públicos.

Para verificar a prevalência de quedas, utilizou-se um questionário com as seguintes perguntas: a) "No último ano, a senhora caiu alguma vez?". Para as participantes que responderam positivamente perguntou-se: b) "Em alguma dessas quedas ocorreu uma fratura?" Em caso de resposta afirmativa, foi perguntado: c) "Qual parte do corpo foi fraturada?" Esse questionário foi adotado conforme estudo anterior ${ }^{19}$.

\section{Avaliação antropométrica}

Para a avaliação antropométrica, o índice de massa corporal (IMC) foi calculado por meio da massa corporal (quilogramas) dividido pela estatura (metro) ao quadrado (IMC = massa corporal/ estatura $^{2}$ ). Para medir a massa corporal, utilizou-se uma balança (Filizola ${ }^{\circledR}$, modelo 31, São Paulo, Brasil), apresentando precisão de $0,1 \mathrm{~kg}$. A participante foi posicionada descalça no centro da plataforma, com o peso igualmente distribuído sobre os dois pés e vestindo apenas camiseta e shorts ou calça de ginástica. Para medir a estatura, foi usado um estadiômetro, com precisão $0,01 \mathrm{~m}$, fixado à balança (Filizola $\Re$, modelo 31, São Paulo, Brasil). A participante posicionou-se ereta, com os pés unidos, a cabeça orientada olhando para frente e foi orientada a inspirar no momento da medida. Todas as medidas foram feitas seguindo os protocolos da Sociedade Internacional de Cineantropometria ${ }^{20}$.

\section{Avaliação da aptidão física}

Para avaliação da aptidão física, foram realizados os seguintes testes motores validados e com a reprodutibilidade determinada ${ }^{14,21-23}$ : (i) Flexibilidade do tronco e membros inferiores 
- teste de sentar e alcançar: com a participante sentada em uma cadeira foi realizada a medida da distância entre a extremidade distal do dedo médio da mão e a extremidade distal do terceiro dedo do pé; (ii) Flexibilidade membros superiores - teste de alcançar atrás das costas: foi medida a distância entre as extremidades distais dos dois dedos médios das mãos, sendo que a mão que foi por cima, a palma encostou às costas e a mão que foi por baixo, o dorso encostou às costas. Em ambos os testes de flexibilidade, as participantes escolheram o lado a ser avaliado. Foram realizadas duas tentativas, sendo considerado o melhor resultado; (iii) Resistência de força de membros inferiores teste de sentar e levantar: maior número de repetições em que a participante sentou e levantou de uma cadeira durante $30 \mathrm{~s}$; (iv) Resistência de força de membros superiores - flexão e extensão de cotovelo sentada em uma cadeira: maior número de repetições em $30 \mathrm{~s}$ que a participante, com um halter de $2,27 \mathrm{~kg}$, partiu da posição com o cotovelo estendido e o flexionou logo em seguida. Em ambos os testes de força, realizou-se apenas uma tentativa; ( $v$ ) Capacidade aeróbia: seis minutos de caminhada por fora de um retângulo de $4,57 \mathrm{~m}$ por $18,28 \mathrm{~m}$, onde a participante andou o mais rápido possível, sem correr. A distância percorrida foi anotada; (vi) Agilidade e equilíbrio dinâmico: a participante se levantou de uma cadeira e deu uma volta em um cone localizado à direita a $1,5 \mathrm{~m}$ para trás e 1,8 m para o lado da cadeira e retornou à posição sentada na cadeira. Imediatamente, a participante levantou os pés do chão (para garantir a finalização do movimento) e iniciou o mesmo movimento do lado oposto, completando um ciclo. Uma tentativa equivaleu a dois ciclos. Foram realizadas duas tentativas e o resultado final foi o menor tempo na realização entre as duas tentativas.

\section{Análise estatística}

Inicialmente realizou-se uma descrição dos dados utilizando categorias para caracterizar a amostra. Os dados estão expressos em termos de média e desvio padrão.

O Teste $t$ para amostras independentes foi realizado para comparar os grupos relativos às quedas (presença ou ausência no último ano). A Análise de Variância com covariáveis (ANCOVA) foi utilizada para comparar os grupos de acordo com as modalidades de exercícios físicos (treinamento combinado, treinamento funcional e treinamento multimodal). As covariáveis utilizadas foram a idade, o IMC e o tempo de prática de exercícios físicos. Vale ressaltar que um dos pré-requisitos para utilização da ANCOVA é que a variável independente não apresente efeito nas covariáveis e para checar este aspecto foi realizada uma Análise de Variância (ANOVA). Estes testes têm como pressupostos que os resíduos (ei) tenham distribuição normal e variância constantes, sendo que estes pressupostos foram checados e validados ${ }^{24}$. As análises foram realizadas considerando-se um nível de significância de $5 \%$ utilizando-se o programa SPSS (versão 26 ).

\section{RESULTADOS}

Este estudo foi realizado predominantemente com idosas jovens (60 a 69 anos), com ensino superior completo e classificação socioeconômica na classe B. Conforme desenho do estudo, todas as participantes praticavam alguma modalidade de exercícios físicos (treinamentos combinado, funcional e multimodal). No entanto, a maioria praticava exercício físico há mais de um ano ou há mais de 10 anos. Quanto ao IMC, as participantes apresentaram principalmente sobrepeso ou peso normal. Em relação à hipertensão, diabetes e dislipidemias, a maioria das participantes não apresentaram essas doenças. Por outro lado, as participantes que apresentaram, realizam tratamento com medicamento (Tabela 1 ).

O grupo sem a ocorrência de quedas no último ano em comparação ao grupo com a ocorrência de quedas apresentou menor idade $[\mathrm{t}(42)=-2,641$; $\mathrm{p}<0,05]$ e maior tempo de prática de exercícios físicos $[\mathrm{t}(42)=2,070 ; \mathrm{p}<0,05]$, além de melhores resultados nos testes de flexibilidade de sentar e alcançar $[\mathrm{t}(42)=2,520 ; \mathrm{p}<0,05]$ e mãos nas costas $[\mathrm{t}(42)=2,193 ; \mathrm{p}<0,05]$, nos testes de força de flexão de cotovelo $[\mathrm{t}(42)=2,189 ; \mathrm{p}<0,05]$ e de sentar e levantar $[t(42)=2,077 ; p<0,05]$, bem como no teste de caminhada $6 \min [\mathrm{t}(42)=2,807$; $p<0,05]$. Não houve diferença estatística entre os grupos no teste de agilidade e equilíbrio dinâmico $[\mathrm{t}(42)=0,203 ; \mathrm{p}>0,05]$ e no IMC $[\mathrm{t}(42)=0,410$; $p>0,05]$ (Tabela 2). 


\section{Tabela 1}

Características socioeconômicas, demográficas e relacionadas ao nível habitual de atividade física das participantes

\begin{tabular}{lcc}
\hline Variável & $(\mathbf{n}=\mathbf{4 4 )}$ & \% \\
\hline Idade & & \\
50 a 59 anos & 11 & 25,0 \\
60 a 69 anos & 24 & 54,5 \\
70 a 80 anos & 9 & 20,5 \\
\hline Escolaridade & & \\
\hline Superior Completo & 20 & 45,5 \\
Superior Incompleto & 2 & 4,5 \\
Médio Completo & 11 & 25 \\
Fundamental Completo & 4 & 9,1 \\
Fundamental Incompleto & 7 & 15,9 \\
\hline Classificação Econômica & & \\
\hline A2 & 6 & 13,6 \\
B1 & 12 & 27,3 \\
B2 & 16 & 36,5 \\
C1 & 6 & 13,6 \\
C2 & 2 & 4,5 \\
D & 2 & 4,5 \\
\hline Tempo de prática de & & \\
\hline
\end{tabular}

Tempo de prática de exercícios

\begin{tabular}{lcc}
\hline Entre 6 meses e 1 ano & 11 & 25 \\
Entre 1 e 10 anos & 17 & 38,6 \\
Mais de 10 anos & 16 & 36,4 \\
\hline Classificação IMC & & \\
\hline Peso normal & 17 & 38,6 \\
Sobrepeso & 18 & 40,9 \\
Obesidade & 9 & 20,5 \\
\hline Hipertensão & & \\
\hline Não & 27 & 61,4 \\
Sim e usa medicamento & 17 & 38,6 \\
\hline Diabetes & & \\
\hline Não & 38 & 86,3 \\
Sim e usa medicamento & 6 & 13,7 \\
\hline Dislipidemia & & \\
\hline Não & 31 & 70,5 \\
Sim e usa medicamento & 13 & 29,5 \\
\hline MC indice de massa corpor & & \\
\hline
\end{tabular}

IMC - índice de massa corporal

\section{Tabela 2}

Comparação de grupos (quedas) em relação à idade, tempo de prática de exercícios e aptidão física

\begin{tabular}{|c|c|c|c|}
\hline & \multicolumn{2}{|c|}{ Quedas } & \multirow[b]{2}{*}{ Valor $\mathbf{p}$} \\
\hline & $\begin{array}{c}\text { Não } \\
(n=36)\end{array}$ & $\underset{(n=8)}{\operatorname{Sim}}$ & \\
\hline Idade (anos) & $\begin{array}{c}63,9 \\
(7)\end{array}$ & $\begin{array}{l}71,5 \\
(9,2)\end{array}$ & 0,012 \\
\hline Tempo de Prática (anos) & $\begin{array}{c}10,3 \\
(11,5)\end{array}$ & $\begin{array}{c}5,3 \\
(4,1)\end{array}$ & 0,046 \\
\hline IMC $\left(\mathrm{kg} / \mathrm{m}^{2}\right)$ & $\begin{array}{l}28,6 \\
(4,7)\end{array}$ & $\begin{array}{c}27,9 \\
(4)\end{array}$ & 0,684 \\
\hline Sentar e Alcançar (cm) & $\begin{array}{c}6,3 \\
(8,7)\end{array}$ & $\begin{array}{c}-3,5 \\
(14,6)\end{array}$ & 0,016 \\
\hline Mãos nas Costas (cm) & $\begin{array}{l}-2,8 \\
(9)\end{array}$ & $\begin{array}{c}-10,9 \\
(12,1)\end{array}$ & 0,034 \\
\hline Flexão Cotovelo (reps) & $\begin{array}{l}16,8 \\
(4,1)\end{array}$ & $\begin{array}{l}13,3 \\
(4,1)\end{array}$ & 0,034 \\
\hline Sentar e Levantar (reps) & $\begin{array}{l}16,4 \\
(4,5)\end{array}$ & $\begin{array}{l}13,4 \\
(4,5)\end{array}$ & 0,046 \\
\hline Caminhada 6 min $(m)$ & $\begin{array}{l}561,1 \\
(75,7)\end{array}$ & $\begin{array}{l}473,9 \\
(96,6)\end{array}$ & 0,008 \\
\hline Agilidade e Equilíbrio (s) & $\begin{array}{c}25 \\
(6,8)\end{array}$ & $\begin{array}{l}24,5 \\
(6,1)\end{array}$ & 0,840 \\
\hline
\end{tabular}

É possível observar na Tabela 3 que não houve diferença entre os grupos, de acordo com as diferentes modalidades de treinamento para idade $[F(2,41)=1,216 ; p>0,05]$, tempo de prática de exercícios $[F(2,41)=0,383 ; p>0,05]$ e $\operatorname{IMC}[F(2,41)=0,207 ; p>0,05]$. Também não houve diiferença estatística entre os grupos após o controle das covariáveis idade, tempo de prática e IMC nos testes de flexibilidade de sentar e alcançar $[F(2,38)=0,940 ; p>0,05]$ e mãos nas costas $[F(2,38)=0,383 ; p>0,05]$, testes de força de flexão de cotovelo $[F(2,38)=0,153 ; p>0,05]$ e sentar e levantar $[F(2,38)=1,018 ; p>0,05]$, caminhada $[F(2,38)=1,592 ; p>0,05]$ e agilidade e equilíbrio dinâmico $[F(2,38)=1,941 ; p>0,05]$. Além disso, é importante ressaltar que as participantes que apresentaram queda no último ano $(n=8)$ apareceram distribuídas de forma homogênea nos grupos: treinamento combinado $(n=3)$, treinamento funcional $(n=2)$ e treinamento multimodal $(n=3)$. 


\section{Tabela 3}

Comparação de grupos (diferentes tipos de treinamento) em relação à idade, tempo de prática de exercícios e aptidão física

\begin{tabular}{lcccc}
\hline & $\begin{array}{c}\text { Treinamento } \\
\text { Combinado } \\
(\mathbf{n}=\mathbf{1 3})\end{array}$ & $\begin{array}{c}\text { Treinamento } \\
\text { Funcional } \\
(\mathbf{n = 1 7 )}\end{array}$ & $\begin{array}{c}\text { Treinamento } \\
\text { Multimodal } \\
\text { (n= 14) }\end{array}$ & Valor p \\
\hline Idade (anos) & $66,4(6,3)$ & $62,9(8,8)$ & $67(7,9)$ & 0,307 \\
Tempo de Prática (anos) & $8,2(8,2)$ & $7,6(12,2)$ & $10,7(10,6)$ & 0,684 \\
IMC (kg/m²) & $26,2(3,8)$ & $26,8(5,1)$ & $26,8(3,9)$ & 0,814 \\
Sentar e Alcançar (cm) & $3,9(11,5)$ & $4(10,6)$ & $5,7(10,2)$ & 0,940 \\
Mãos nas Costas (cm) & $3,2(8,7)$ & $3(11,5)$ & $4,9(9,3)$ & 0,383 \\
Flexão Cotovelo (reps) & $15,3(3,3)$ & $16,9(3,9)$ & $16(5,6)$ & 0,859 \\
Sentar e Levantar (reps) & $14,9(5,8)$ & $15,1(3,7)$ & $17,5(9,4)$ & 0,371 \\
Caminhada 6 min (m) & $538,2(85,8)$ & $566,9(81,8)$ & $525,6(90,3)$ & 0,217 \\
Agilidade e Equilíbrio (s) & $26,5(9,1)$ & $23,1(3,6)$ & $25,8(6,6)$ & 0,158 \\
\hline
\end{tabular}

Média (DP). IMC - índice de massa corporal; cm - centímetros; reps - repetições; $s$ - segundos; $m$ - metros.

\section{DISCUSSÃO}

No presente estudo verificou-se uma pior aptidão física no grupo de mulheres que apresentou ocorrência de quedas no último ano. Este grupo apresentou piores resultados nos testes de flexibilidade, força de membros superiores e de membros inferiores, bem como capacidade aeróbia. Além disso, este grupo apresentou maior idade e menor tempo de prática de exercícios físicos (Tabela 2). Estes resultados sugerem que o envelhecimento associado ao comportamento sedentário ao longo da vida pode apresentar relação com o baixo nível de aptidão física e o aumento da ocorrência de quedas. Assim, destaca-se a importância de bons níveis de aptidão física como ação de prevenção e reabilitação das quedas.

O envelhecimento é marcado por um declínio funcional do organismo, incluindo alterações no equilíbrio e redução de massa muscular com consequente diminuição de força e resistência muscular. Por outro lado, o exercício físico é considerado uma potente ferramenta para manutenção dessas capacidades motoras, proporcionando o aumento da massa e força muscular, reduzindo o risco de quedas $s^{2,3,25}$. Nesse sentido, uma atenção importante deve ser oferecida ao estilo de vida ativo ao longo da vida já que mudanças podem acontecer com o passar dos anos. Indivíduos com idade mais elevada podem apresentar menores níveis de atividade física, conforme dados nacionais que mostraram que entre 20 e $30 \%$ das mulheres com idade acima de 55 anos apresentaram atividade física no tempo livre de 150 min semanais moderadas ou 75 minutos vigorosas, enquanto nos grupos etários mais jovens estes números se aproximaram de $40 \%{ }^{26}$.

Diversos estudos têm destacado as quedas dentre os problemas de Saúde Pública relacionados ao envelhecimento devido às complicações que promovem, como aumento dos gastos em saúde e limitações físicas e funcionais que levam à dependência e risco de morte ${ }^{1,5,6}$. Nesse sentido, aumentar a aptidão física parece ser uma estratégia importante na prevenção e redução de quedas, bem como na reabilitação no contexto pós-queda, tanto nos aspectos físicos como também psicológicos, que envolvem a confiança e a retomada das AVDs de forma independente, com consequente benefício para o indivíduo e o Sistema Público de Saúde. O equilíbrio das capacidades motoras com boas funções do sistema nervoso proporcionará ao idoso desempenhar as AVDs de forma segura e eficaz ${ }^{3,7}$.

Neste estudo, não houve diferença entre as modalidades de exercícios físicos (treinamentos combinado, funcional e multimodal) praticados pelas participantes para nenhuma das variáveis relacionadas à aptidão física analisadas, i.e., IMC, testes de flexibilidade, força, resistência aeróbia e agilidade e equilíbrio dinâmico (Tabela 3). Em relação aos testes motores, é importante destacar que os resultados observados sugerem benefícios 
funcionais muito bons para as participantes do estudo, já que ao comparar com os valores normativos disponíveis na literatura, de acordo com a faixa etária das participantes, observam-se estes resultados nos percentis mais elevados, sempre acima de 5014,22,23.

A análise dos resultados relacionados ao IMC foi realizada seguindo as recomendações do Sistema de Vigilância Alimentar e Nutricional (SISVAN) que adota pontos de corte para os idosos, considerando as alterações na composição corporal próprias do envelhecimento. Observou-se que a média apresentada pelas participantes na Tabela 3 nos três grupos de treinamento, apesar de muito próximo da faixa de sobrepeso, está na faixa de classificação para eutrofia (peso normal - entre 22 e $27 \mathrm{~kg} / \mathrm{m}^{2}$ ) 27 . De acordo com os resultados dos testes motores e IMC, observa-se que estas modalidades de exercícios físicos mais globais podem ser consideradas importantes para a manutenção de bons níveis de aptidão física no contexto do envelhecimento.

Além de apresentar bons resultados na aptidão física, as prevalências de sobrepeso, obesidade, hipertensão, diabetes e dislipidemias das participantes do presente estudo (Tabela 1) estão abaixo das prevalências atuais do Brasil26,28. Estes resultados podem estar relacionados à prática regular de exercícios físicos. Foi possível observar sobrepeso e obesidade em 40,9 e $20,5 \%$ das participantes, enquanto os dados nacionais apontam prevalências entre 62 e $66 \%$ para excesso de peso e 25 e $30 \%$ para obesidade, para mulheres nos grupos etários acima de 50 anos. Em relação à hipertensão, diabetes e dislipidemia, no presente estudo a resposta foi afirmativa em $38,6 \%, 13,7 \%$ e $29,5 \%$ das participantes, enquanto os dados nacionais apontam para prevalências entre 51 e $64 \%, 16$ e $22 \%{ }^{26}$ e 43 e $45 \%{ }^{28}$, respectivamente.

A importância das propostas globais de intervenção de exercícios físicos no contexto do enveIhecimento vem sendo discutida nos últimos anos por meio de posicionamento oficiais e estudos de intervenção ${ }^{6,9,11,13,29}$. Ressalta-se que as alterações físicas e fisiológicas relacionadas ao envelhecimento acometem diversas capacidades e habilidades motoras e não somente uma específica.

Estudos anteriores do nosso grupo de pesquisa apresentaram informação neste sentido. Observou-se que os grupos que realizavam "caminhada e mais um tipo de exercício físico" e "caminhada e mais dois ou mais tipos de exercícios" apresentaram melhores resultados na aptidão física e variáveis relacionadas à saúde cardiovascular do que os grupos que praticavam "apenas caminhada" ou era "inativo fisicamente" ${ }^{130}$. Ainda, a saúde cardiovascular apresentou um maior número de associações na análise com a aptidão física do que com o nível habitual de atividade física ${ }^{15}$, destacando a importância de aspectos mais globais nas intervenções de exercícios físicos, voltados para a aptidão física, conforme apresentados no presente estudo.

Alguns estudos utilizaram estratégias mais globais como proposta de intervenção. Como exemplo, Neves et al. ${ }^{13}$ compararam o efeito de oito semanas de diferentes modalidades de treinamento, incluindo o combinado, funcional e multimodal na aptidão física de mulheres adultas - os resultados apontaram para melhoras em todas as modalidades de treinamento, entretanto com diferentes componentes da aptidão física de acordo com a modalidade. Ainda, o estudo de Resende Neto ${ }^{29}$ que ao comparar o efeito de 12 semanas de treinamento funcional com o treinamento de força na aptidão física de idosos pré-frágeis, encontrou melhora nos seis testes realizados somente no grupo treinamento funcional.

Uma limitação do presente estudo é que os dados a partir da análise transversal não permitem o estabelecimento da relação de causa e efeito. Entretanto, ainda assim, é possível sugerir que a ocorrência das quedas pode ter levado a alguma limitação em relação à aptidão física das participantes, ou que a baixa aptidão física pode ter sido um fator facilitador para as quedas. Outra limitação que pode ser apontada é o tamanho amostral $(n=44)$, entretanto é importante ressaltar que a amostra do presente estudo é bastante específica: somente mulheres com idade entre 50 e 80 anos e que praticavam há pelo menos seis meses uma das três modalidades de treinamento (combinado, funcional ou multimodal). Observaram-se poucas possibilidades de prática neste sentido para esta população em Ribeirão Preto, SP.

A partir dos piores resultados na aptidão física no grupo que apresentou ocorrência de queda, sugere-se como aplicação prática o desenvolvimento de estratégias que possam manter bons níveis de aptidão física na população adulta e 
idosa, para prevenir as quedas e oferecer atenção na recuperação do pós-queda. Com a queda, pode acontecer a diminuição da atividade física causada pelo medo e insegurança, com consequências negativas para a independência nas AVDs e vida social. Ressalta-se ainda, as implicações das quedas para o Sistema de Saúde, destacando a importância que a prevenção e a recuperação pós-queda podem apresentar para evitar uma maior demanda nos níveis secundário e terciário. E, como não foi encontrada diferença entre os grupos para nenhuma das variáveis analisadas, sugere-se que os três diferentes tipos de treinamento (combinado, funcional e multimodal), vistos como mais globais, são estratégias importantes e que estão relacionadas a bons níveis de aptidão física, já que na comparação com os valores normativos disponíveis na literatura para cada uma das variáveis relacionadas à aptidão física, os resultados foram sempre satisfatórios. Portanto, sugere-se estas modalidades de exercícios físicos como opções importantes e adequadas para as intervenções de atividade física em adultos e idosos.

Dentre os apontamentos futuros, a realização de estudos transversais em tamanhos amostrais maiores, bem como estudos de intervenção utilizando estas diferentes modalidades de exercícios físicos, ambos em populações com diferentes características, poderão fortalecer o conhecimento sobre o tema. Ainda, destaca-se a importância do oferecimento de exercícios físicos para os adultos idosos, com maior atenção para estas propostas mais globais e na supervisão de um profissional de Educação Física, responsável por uma prática segura e adequada ao perfil de cada indivíduo.

\section{CONCLUSÃO}

Foi possível observar piores resultados na aptidão física das participantes que apresentaram ocorrência de queda no último ano, bem como maior idade e menor tempo de prática de exercícios; e, após o controle das covariáveis idade, tempo de prática de exercícios e IMC, não foi possível verificar diferença entre os grupos de acordo com as diferentes modalidades de treinamento (combinado, funcional e multimodal) para nenhuma das variáveis relacionadas à aptidão física. Entretanto, ressalta-se que os três grupos apresentaram bons níveis de aptidão física, de acordo com os valores normativos disponíveis na literatura na aptidão física. Desta forma, observa-se que estas modalidades com propostas mais globais de treinamento são importantes para a manutenção de bons níveis de aptidão física e podem estar relacionadas com a prevenção e reabilitação das quedas em mulheres com idade entre 50 e 80 anos.

\section{REFERÊNCIAS}

1. Maresova $\mathrm{P}$, Javanmardi E, Barakovic $\mathrm{S}$, Barakovic Husic J, Tomsone S, Krejcar O, et al. Consequences of chronic diseases and other limitations associated with old age a scoping review. BMC public health. 2019;19(1):1431. doi: $10.1186 / s 12889-019-7762-5$

2. Tomas MT, Galan-Mercant A, Carnero EA, Fernandes B. Functional Capacity and Levels of Physical Activity in Aging: A 3-Year Follow-up. Frontiers in medicine. 2017;4:1-8. doi: 10.3389/fmed.2017.00244

3. Zhuang J, Huang $L$, Wu $Y$, Zhang $Y$. The effectiveness of a combined exercise intervention on physical fitness factors related to falls in community-dwelling older adults. Clinical interventions in aging. 2014;9:131-40. doi: $10.2147 /$ cia.S56682

4. James SL, Lucchesi LR, Bisignano C, Castle CD, Dingels ZV, Fox JT, et al. The global burden of falls: global, regional and national estimates of morbidity and mortality from the Global Burden of Disease Study 2017. Injury prevention : journal of the International Society for Child and Adolescent Injury Prevention. 2020. doi: 10.1136/injuryprev-2019-043286

5. Trevisan C, Rizzuto D, Maggi S, Sergi G, Wang HX, Fratiglioni $L$, et al. Impact of Social Network on the Risk and Consequences of Injurious Falls in Older Adults. Journal of the American Geriatrics Society. 2019;67(9):1851-58. doi: 10.1111/jgs.16018

6. Moncada LVV, Mire LG. Preventing Falls in Older Persons. American family physician [Internet]. 2017 Aug 15; 96(4):[240-7 pp.]. Disponível em: https://www.aafp. org/afp/2017/0815/p240.html

7. Chase JD, Phillips LJ, Brown M. Physical Activity Intervention Effects on Physical Function Among Community-Dwelling Older Adults: A Systematic Review and Meta-Analysis. J Aging Phys Act. 2017;25(1):14970. doi: 10.1123/japa.2016-0040

8. Caspersen $\mathrm{CJ}$, Powell KE, Christenson GM. Physical activity, exercise, and physical fitness: definitions and distinctions for health-related research. Public health reports (Washington, DC : 1974) [Internet]. 1985; 100(2):[126-31 pp.]. Disponível em: https://www.ncbi. nlm.nih.gov/pmc/articles/PMC1424733/pdf/pubhealthrep00100-0016.pdf 
9. Chodzko-Zajko WJ, Proctor DN, Fiatarone Singh MA, Minson CT, Nigg CR, Salem GJ, et al. American College of Sports Medicine position stand. Exercise and physical activity for older adults. Medicine and science in sports and exercise. 2009;41(7):1510-30. doi: 10.1249/ MSS.0b013e3181a0c95c

10. Nelson ME, Rejeski WJ, Blair SN, Duncan PW, Judge JO, King $A C$, et al. Physical activity and public health in older adults: recommendation from the American College of Sports Medicine and the American Heart Association. Medicine and science in sports and exercise. 2007;39(8):1435-45. doi: $10.1249 / \mathrm{mss} .0 \mathrm{~b} 013 \mathrm{e} 3180616 \mathrm{aa} 2$

11. Precoma DB, Oliveira GMM, Simao AF, Dutra OP, Coelho OR, Izar MCO, et al. Updated Cardiovascular Prevention Guideline of the Brazilian Society of Cardiology - 2019. Arquivos brasileiros de cardiologia. 2019;113(4):787891. doi: 10.5935/abc.20190204

12. Nascimento R, Kanitz A, Kruel F. Efeitos de diferentes estratégias de treinamento combinado na força muscular e na potência aeróbia de idosos: uma revisão sistemática. Rev bras ativ fís saúde. 2015;20(4):329-39. doi: $10.12820 /$ rbafs.v.20n4p329

13. Neves LM, Diniz TA, Rossi FE, Fortaleza ACS, Horimoto ET, Geraldo VO, et al. The effect of different training modalities on physical fitness in women over 50 years of age. Motriz: J Phys Ed. 2016;22(4):319-26. doi: $10.1590 /$ s1980-6574201600040016

14. Rikli R, Jones C. Development and validation of criterion-referenced clinically relevant fitness standards for maintaining physical independence in later years. The Gerontologist. 2013;53(2):255-67. doi: 10.1093/ geront/gns071

15. Trapé AA, Lizzi EAS, Jacomini AM, Hott SC, Bueno Júnior $\mathrm{CR}$, Zago AS. Aptidão física e nível habitual de atividade física associados à saúde cardiovascular em adultos e idosos. Medicina (Ribeiräo Preto). 2015;48(5):457-66. doi: $10.11606 /$ issn.2176-7262.v48i5p457-466

16. Matsudo SM, Matsudo VKR, Araújo TL. Perfil do nível de atividade física e capacidade funcional de mulheres maiores de 50 anos de idade de acordo com a idade cronológica. Rev bras ativ fís saúde. 2001;6(1):12-24. doi: $10.12820 /$ rbafs.v.6n1p12-24

17. ABEP. Associação Brasileira de Empresas de Pesquisa. Critério de Classificação Econômica Brasil. 2018.

18. Kamakura W, Mazzon JA. Critérios de estratificação e comparação de classificadores socioeconômicos no Brasil. Rev adm empres. 2016;56. doi: 10.1590/ S0034-759020160106

19. Siqueira FV, Facchini LA, Piccini RX, Tomasi E, Thume E, Silveira DS, et al. Prevalence of falls and associated factors in the elderly. Revista de saude publica. 2007;41(5):749-56. doi: 10.1590/ s0034-89102007000500009

20. Stewart A, Marfell-Jones $M$, Olds $T$, Ridder $H$. International standards for anthropometric assessment. Lower Hutt: ISAK; 2001.

21. Osness WH, Adrian M, Clark B, Hoeger W, Raab D, Wiswell R. Functional fitness assessment for adults over 60 years (A field based assessment): Published test protocols. . Reston: American Alliance of Health, Physical Education, Recreation and Dance (AAHPERD); 1990.

22. Rikli R, Jones C. Development and Validation of a Functional Fitness Test for Community-Residing Older Adults. JAPA. 1999;7(2):129-61. doi: 10.1123/ japa.7.2.129

23. Rikli R, Jones $\mathrm{C}$. Teste de aptidão física para idosos. Barueri: Manole; 2008.

24. Montgomery DC. Design and analysis of experiments. New Jersey: John Wiley \& Sons; 2017.

25. Fisher JP, Steele J, Gentil P, Giessing J, Westcott WL. A minimal dose approach to resistance training for the older adult; the prophylactic for aging. Experimental gerontology. 2017;99:80-6. doi: 10.1016/j.exger.2017.09.012

26. Brasil. Ministério da Saúde. Vigilância de Fatores de Risco para doenças crônicas não transmissíveis (VIGITEL) 2018. Brasília: Ministério da Saúde; 2019.

27. Brasil. Ministério da Saúde. Secretaria de Atenção à Saúde. Departamento de Atenção Básica. Orientações para a coleta e análise de dados antropométricos em serviços de saúde: norma técnica do sistema de vigilância alimentar e nutricional - SISVAN. Brasília: Ministério da Saúde; 2011.

28. Brasil. Ministério da Saúde. Vigilância de Fatores de Risco para doenças crônicas não transmissíveis (VIGITEL) 2016. Brasília: Ministério da Saúde; 2017.

29. Resende Neto AG, Feitosa Neta ML, Santos MS, Teixeira CVS, Sá CA, Silva-Grigoletto ME. Treinamento funcional versus treinamento de força tradicional: efeitos sobre indicadores da aptidão física em idosas pré-frageis. Motricidade [Internet]. 2016; 12(S2):[44-53 pp.]. Disponível em: https://revistas.rcaap.pt/motricidade/ article/view/9790/9155

30. Trapé AA, Sacardo AL, Cássia AF, Monteiro HL, Zago AS. Relação entre a prática da caminhada não supervisionada e fatores de risco para as doenças cardiovascular em adultos e idosos. Medicina (Ribeiräo Preto). 2014;47(2):165-76. doi: 10.11606/issn.2176-7262. v47i2p165-176 